\title{
Combined phacocanaloplasty for open-angle glaucoma and cataract: 12 months results
}

This article was published in the following Dove Press journal:

Clinical Ophthalmology

\author{
Mahmoud Mohamed \\ Genaidy \\ Hosny Ahmed Zein \\ Ahmed Mostafa Eid \\ Department of Ophthalmology, \\ Faculty of Medicine, Minia University, \\ Minia, Egypt
}

Purpose of the study: To evaluate efficacy, safety, and success rates of canaloplasty combined with phacoemulsification and intraocular lens implantation in patients with open-angle glaucoma (OAG) and visually significant cataract.

Patients and methods: A prospective interventional noncomparative case series carried out in Minia University Hospital from April 2015 to October 2016 on 20 eyes of 18 patients who had visually significant cataract and primary OAG. All the cases had combined phacocanaloplasty. Preoperative best-corrected visual acuity, intraocular pressure (IOP), and number of antiglaucoma medications were collected and compared to postoperative levels, and complications rates were recorded.

Results: 20 eyes of 18 patients ( 5 males and 15 females), with a mean age 57.6 years (range 48-69 years), underwent phacocanaloplasty. Preoperative mean IOP was $25.20 \pm 1.009 \mathrm{mmHg}$. Postoperative IOP decreased to a mean of $14.20 \pm 0.9,14.85 \pm 0.8$, and $15.85 \pm 0.7 \mathrm{mmHG}$ at 3,6 , and 12 months, respectively, with $37 \%$ reduction from preoperative IOP level at one year follow-up visit $(P=0.0005)$. The number of antiglaucoma medications dropped from mean of 1.55 preoperatively to 0.35 postoperatively. LogMAR of best-corrected visual acuity improved from $0.6950 \pm 0.07$ preoperatively to $0.3670 \pm 0.056,0.3460 \pm 0.056$, and $0.03370 \pm 0.052$ at 3,6 , and 12 months postoperatively ( $P=0.0005$ ). Complications were limited to mild hyphemia (one case), mild corneal edema (one case), and mild inflammatory membrane (one case) that resolved in the first week after surgery.

Conclusion: Canaloplasty combined with clear corneal phacoemulsification and intraocular lens implantation may be a safe and effective procedure to lower IOP in adult patients with $\mathrm{OAG}$ and visually significant cataract.

Keywords: cataract, glaucoma, phacoemulsification, canaloplasty

\section{Introduction}

The main goal of glaucoma treatment is reduction of intraocular pressure (IOP). The classic treatment algorithm consists of initial medical therapy followed by glaucoma surgery if necessary, with laser therapy sometimes used to fill the gap between medical therapy and surgery. Many variations of this paradigm exist. The most popular glaucoma surgery utilized is the trabeculectomy with the use of Mitomycin C, which redirects aqueous from the anterior chamber to a subconjunctival bleb. ${ }^{1}$ This procedure, while very effective at lowering IOP, is associated with multiple risks and complications. Intraoperative and postoperative risks include hypotony, hyphema, choroidal detachment, and suprachoroidal hemorrhage. The blebs may be associated with bleb leak, bleb encapsulation, bleb dysesthesia, blebitis, and bleb-associated endophthalmitis. ${ }^{2,3}$ 
Such complications have encouraged many glaucoma surgeons to seek out a less invasive IOP-lowering surgery. Recently, circumferential viscodilation and tensioning of Schlemm's canal using a flexible microcatheter (canaloplasty) in combination with deep sclerectomy has raised interest for the treatment of open-angle glaucoma (OAG) in adults. ${ }^{7}$

Combined surgery to treat both glaucoma and cataract has been of interest due to the frequent coincidence of the two diseases in elderly patients. The reduction in surgical trauma from two separate surgical procedures and the high incidence of cataract development after glaucoma surgery are factors supporting the application of a combined procedure in appropriate patients. ${ }^{4-6}$

Clear corneal phacoemulsification and posterior chamber intraocular lens (IOL) implantation can be combined with a nonpenetrating surgical approach involving microcatheter dilation of the entire circumference of Schlemm's canal, in conjunction with placement of a trabecular tensioning suture in a procedure called canaloplasty in adult patients with cataract and $\mathrm{OAG}{ }^{7}$

Although there multiple studies discussing the efficacy and safety of canaloplasty, the data on canaloplasty in combination with phacoemulsification are limited. ${ }^{8,9}$ The aim of this study was to assess efficacy, safety, and success rates of the IOP-lowering effect obtained by canaloplasty combined with phacoemulsification and IOL implantation in patients with $\mathrm{OAG}$ and visually significant cataract.

\section{Patients and methods}

This was a prospective, interventional, noncomparative case series carried out in Minia University Hospital, Minia, Egypt, from April 2015 to October 2016 on 20 eyes of 18 patients who had visually significant cataract and primary OAG.

All study candidates agreed to participate and signed written informed consent. The study was conducted after approval from the ethical committee of Minia University

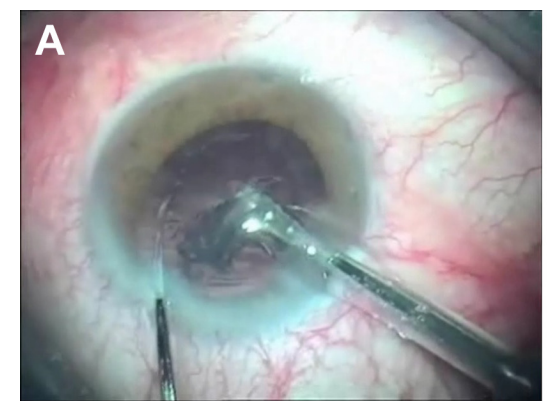

Figure I Clear corneal phacoemulsification (A) and Vicryl 6/0 traction suture (B).
Faculty of medicine. The study meets Declaration of Helsinki and Hospital's Ethical Clearance.

Patients enrolled in the study had visually significant cataract and $\mathrm{OAG}$, no history of previous glaucoma surgery, and an IOP goal in the low-to-mid teens. Exclusion criteria included the following: angle-closure glaucoma (primary or secondary), uveitic glaucoma, angle recession glaucoma, and presence of clear lens. Patients with failed cannulation or complications to penetrating surgery were excluded from the study.

All patients had complete baseline ophthalmic examination before operation that included history of glaucoma, medication use, IOP, best-corrected visual acuity (BCVA), gonioscopy, and slit-lamp and fundus evaluations.

\section{Surgical procedure}

A peribulbar block anesthesia and akinesia were used preoperatively. Phacoemulsification with temporal clear corneal incision and posterior chamber IOL implant were performed on all patients (Figure 1A). We performed phacoemulsification before canaloplasty. The side port and clear corneal incisions were planned in a manner as to not intersect the corneal traction suture that is necessary for canaloplasty. Traction suture with 6.0 vicryl (double pass) was used to expose the site of surgery on the upper sclera, and the eye was tracked inferiorly (Figure 1B).

\section{Canaloplasty technique}

1. Limbal peritomy followed by exposure of bare sclera through clean dissection of conjunctiva and Tenon's capsule was performed, and wet-field cautery was used for hemostasis. No antifibrotics were used.

2. The superficial scleral flap: it is approximately one-third to one-half scleral thickness. We created $5 \times 5 \mathrm{~mm}$ trapezoid flaps and dissected this flap into the clear cornea up to approximately one millimeter in the clear cornea.

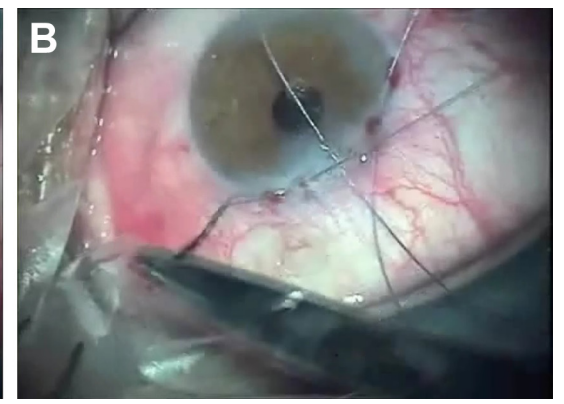



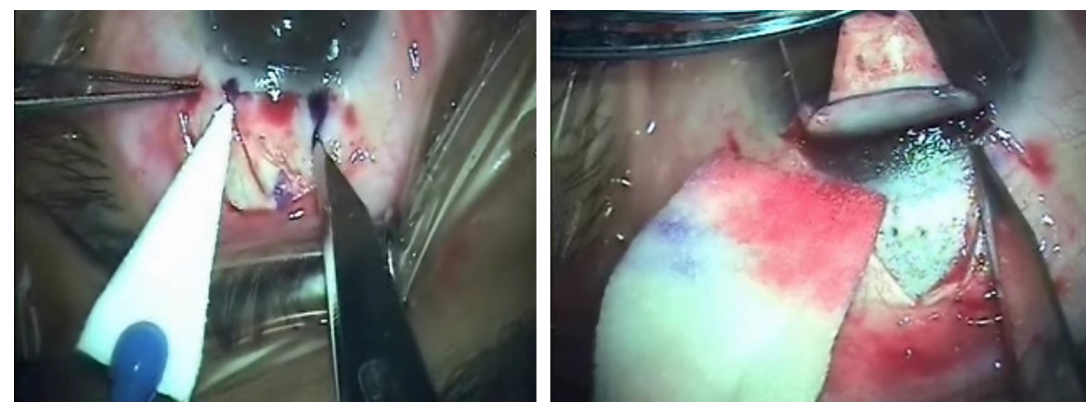

Figure 2 Creation of superficial scleral flap.

This superficial scleral flap was retracted with traction suture (Figure 2).

3. The deep scleral flap: the size was about one-half to one millimeter smaller than the superficial flap. The dissection plane was immediately superficial to the choroid. Choroidal exposure may occur. Anterior dissection of the deep scleral flap can lead to unroofing of the Schlemm's canal. Our anatomical landmark to reach Schlemm's canal was the cross-striations of the scleral spur, as this is immediately anterior (Figure 3).

4. The eye was then decompressed by removing some fluid through the side port in order to decrease the risk of anterior chamber perforation.

5. After releasing pressure in the anterior chamber, we dissected the deep flap anteriorly. We further dissected in this plane past Schwalbe's line to create the descemetic window. Aqueous was seen percolating through the trabecular Descemet's window.

6. Both ends of the canal were dilated using sodium hyaluronate injection (Healon) in the canal. The microcatheter (Glaucolight; DORC International, Zuidland, the Netherlands) was inserted into the canal, and the light of the microscope was dimmed to allow visualization of the illuminating optical fiber through sclera. If resistance was encountered, we retracted the microcatheter and inserted it through the opposite ostium. After successful $360^{\circ}$ cannulation of Schlemm's canal, a 10-0 polypropylene suture (Prolene, Ethicon, Neuchatel, Switzerland) was tied to the tip of the microcatheter which was then retracted, thus introducing the suture throughout the circumference of the canal. The suture was removed from the microcatheter and carefully tied to allow adequate tension on Schlemm's canal while avoiding inadvertent trabeculotomy. The suture tension was done just to allow proper $360^{\circ}$ distention of Schlemm's canal (Figure 4).

7. The deep scleral flap was excised followed by closing the superficial flap in a watertight manner with interrupted 9.0 vicryl sutures (Figure 5 ).

8. Tenon's capsule and conjunctiva were then closed with 8-0 Vicryl at the limbus (Figure 5), and the surgery was concluded with application of antibiotic-steroid ointment.

\section{Postoperative care}

Postoperatively, patients were placed on steroid and fourthgeneration fluoroquinolone eye drops five times per day for 2 weeks. Then, the patient continued only steroid drops for another 2 weeks.

Patients were seen on 1 day, 1 week, 2 weeks, and 1, 3, 6 , and 12 months postoperatively.
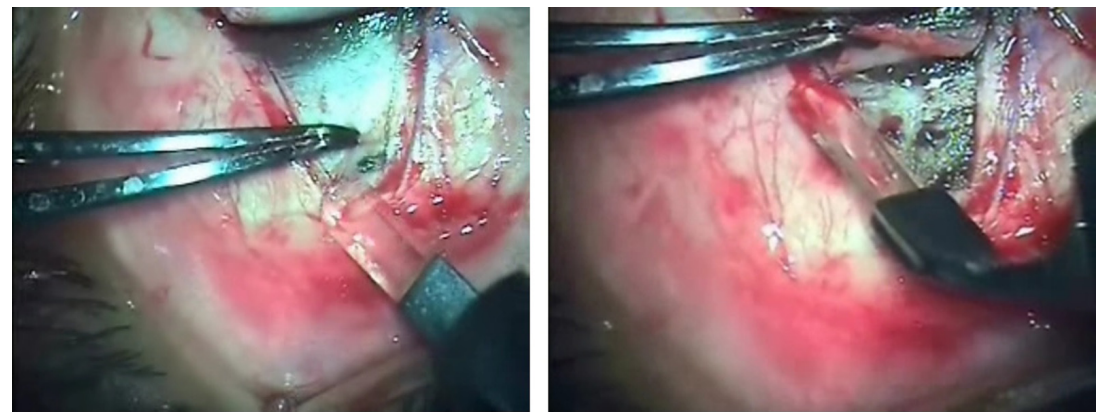

Figure 3 Creation of deep scleral flap. 

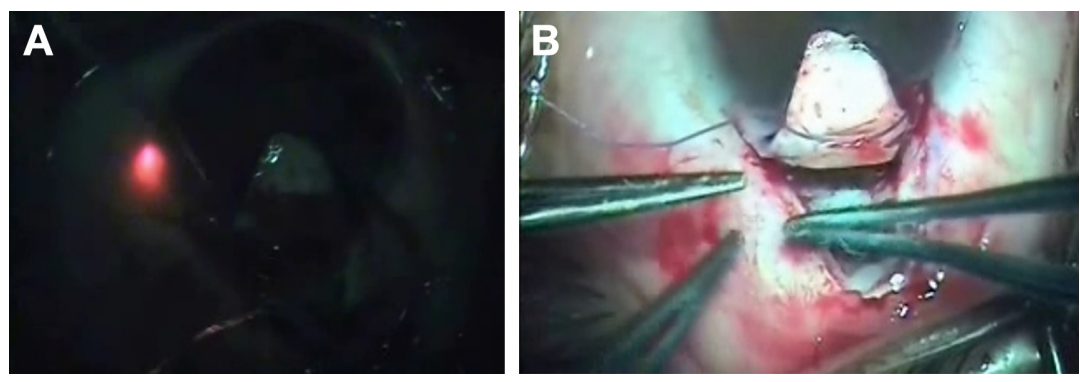

Figure 4 Advancing microcatheter through SC (A) followed by Prolene suture placement (B). Abbreviation: SC, Schlemm's canal.

Data collected included BCVA, IOP, the need for antiglaucoma drugs, and any postoperative complications.

The primary outcomes included mean IOP and mean number of glaucoma medications at the 3-, 6-, and 12-month follow-up visits. Secondary outcomes included the use of glaucoma medications and visual acuity.

\section{Statistical analysis}

Data were collected, coded, and subjected to statistical analysis using SPSS statistical package version 20 (IBM Corp., Armonk, NY, USA). For descriptive statistics, mean and standard deviation (SD) were used to describe quantitative data. Kolmogorov-Smirnov normality test was used to differentiate between parametric data and nonparametric data. For analytical statistics, Wilcoxon signed ranks test was used for comparison of two related parameters preoperatively and postoperatively. $P$-value less than 0.05 was considered statistically significant.

\section{Results}

This was prospective, interventional, noncomparative case series carried out in Minia University Hospital from April 2015 to October 2016 on 20 eyes of 18 patients who had visually significant cataract and primary OAG.
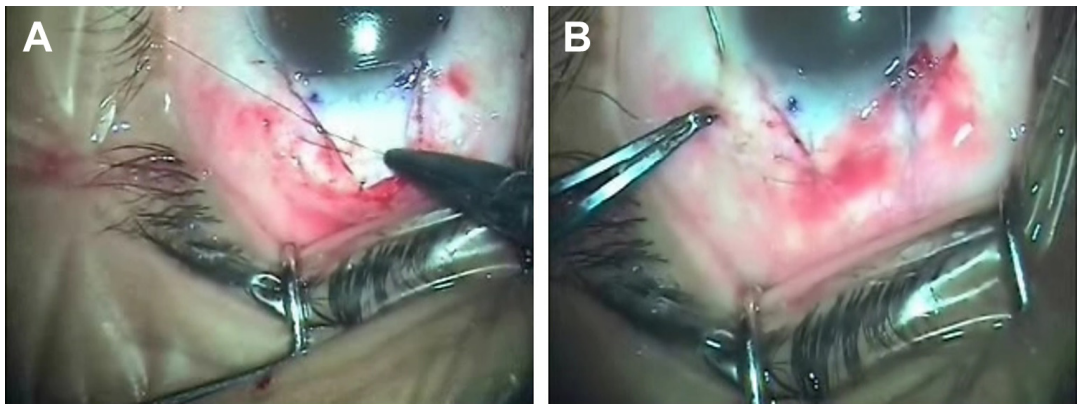

Figure 5 Closure of superficial scleral flap (A) and conjunctiva (B).

There were 5 males (25\%) and 15 females (75\%) with a mean age 57.6 years (range $48-69$ years). The mean preoperative visual acuity was $0.6950 \pm 0.07$ LogMAR, and mean preoperative IOP was $25.20 \mathrm{mmHg} \pm 1.009$ (Table 1).

\section{Changes in IOP}

There was clinically significant reduction in IOP in the postoperative period, where IOP decreased to a mean of $14.20 \pm 0.9,14.85 \pm 0.8$, and $15.85 \pm 0.7 \mathrm{mmHG}$ at 3,6 , and 12 months, respectively, with $37 \%$ reduction from preoperative IOP level at the one year follow-up visit $(P=0.0005)$ (Figure 6 and Table 2). The use of IOP-lowering medications was not discontinued before surgery, and the number of glaucoma medications decreased from a mean of 1.55 (range $0-3$ ) preoperatively to 0.35 postoperatively, and the number of eyes with controlled IOP without antiglaucoma medications reached 12 eyes (60\%) at 12 months postoperatively.

\section{Visual acuity changes}

There was rapid visual recovery in comparison to combined phacotrabeculectomy as this is a blebless operation with no IOP fluctuation postoperatively (Figures 7 and 8). All eyes gained improvement in visual acuity at 12 months postoperatively. LogMAR of BCVA improved 
Table I Patient demographics and baseline characteristics

\begin{tabular}{ll}
\hline & $\begin{array}{l}\text { Combined phaco } \\
\text { and canaloplasty } \\
\mathbf{n}=\mathbf{2 0}\end{array}$ \\
\hline $\begin{array}{l}\text { Gender } \\
\text { Male }\end{array}$ & $5(25 \%)$ \\
Female & $15(75 \%)$ \\
Age (yrs) & $57.6(48-69)$ \\
Mean (range) & \\
No antiglaucoma drugs & $5(25 \%)$ \\
One antiglaucoma drug & $10(50 \%)$ \\
Two antiglaucoma drugs & $4(20 \%)$ \\
Three antiglaucoma drugs & $1(5 \%)$ \\
Mean initial visual acuity & $0.6950 \pm 0.07$ \\
Mean initial IOP, mmHg ( \pm SD) & $25.20 \pm 1.009$ \\
\hline
\end{tabular}

Abbreviations: IOP, intraocular pressure; SD, standard deviation.

from $0.6950 \pm 0.07$ preoperatively to $0.3670 \pm 0.056$, $0.3460 \pm 0.056$, and $0.03370 \pm 0.052$ at 3,6 , and 12 months postoperatively $(P=0.0005)$ (Figure 9 and Table 3 ).

\section{Intraoperative and postoperative complications}

Intraoperatively, we had one case with mild hyphema, and the conjunctiva was not sutured in one irritable patient. As regards the postoperative complications, we had mild corneal edema in 1 eye, mild inflammatory membrane in 1 eye, recessed conjunctiva in 1 eye, mild hyphema in 1 eye, and astigmatism due to tensioning (2DC) in 2 eyes.

\section{Discussion}

Primary OAG and visually significant cataract commonly coexist in elderly people, leading to limitation of daily activities and increasing dependence on antiglaucoma medications, Multiple studies demonstrate that phacoemulsification may produce long-term decrease in IOP in glaucoma patients. ${ }^{10}$ This effect is thought to be produced by

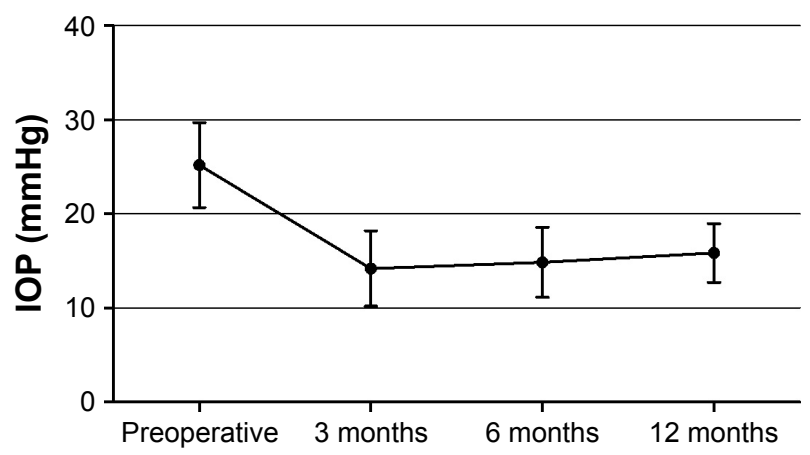

Figure 6 IOP preoperatively and at 3, 6, and 12 months postoperatively. Abbreviation: IOP, intraocular pressure.
Table 2 IOP at baseline, 3, 6, and 12 months posttreatment

\begin{tabular}{ll}
\hline & $\begin{array}{l}\text { Combined phaco } \\
\text { and canaloplasty } \\
\mathbf{n}=\mathbf{2 0}\end{array}$ \\
\hline Preoperative IOP, $\mathrm{mmHg}($ mean $\pm \mathrm{SD})$ & $25.20 \pm \mathrm{I}$ \\
3 months & $14.20 \pm 0.9^{*}$ \\
6 months & $14.85 \pm 0.8^{*}$ \\
12 months & $15.85 \pm 0.7^{*}$ \\
\hline
\end{tabular}

Notes: $* P=0.0005$. There was no statistically significant reduction in IOP from 3,6 to 12 months postoperatively $(P=0.234)$.

three mechanisms: decreased secretion of aqueous humor due to production of free radicals or partial ciliary body detachment, improved uveoscleral outflow due to increased synthesis of endogenous prostaglandins, and improved trabecular outflow due to increased space in the anterior chamber resulting in increased posterior traction on the scleral spur and expansion of the trabecular meshwork and Schlemm's canal (SC), and washing out effect on trabecular meshwork. ${ }^{11}$

Viscodilation of SC using a flexible microcatheter with subsequent suture tensioning is designed to be a blebless procedure that requires no antifibrotic agents and has been suggested to restore the natural outflow system of the eye. ${ }^{9}$ Canaloplasty targets the pathologic site of maximal aqueous outflow resistance in OAG theoretically by stretching SC and adjacent juxtacanalicular outflow system, and a pilocarpinelike effect on the trabecular meshwork (TM) may occur as well. ${ }^{8,9}$ The effect of suture tensioning may actually alter the anatomical structure of the TM to favor aqueous outflow over time. This and/or delayed reopening of distal collector channels has been proposed to offer mild, late IOP reduction remotely after surgery. ${ }^{9}$

So, by combining the two procedures we can get longterm reduction of IOP, improved visual acuity, and decreased number of antiglaucoma medications.

In our case series of 20 eyes of 18 patients we achieved a $37 \%$ reduction in IOP at one year follow-up from the preoperative IOP value, and the number of glaucoma medications decreased from a mean of 1.55 (range $0-3$ ) preoperatively to 0.35 postoperatively, and the number of eyes with controlled IOP without antiglaucoma medications reached 12 eyes (60\%) at 12 months postoperatively.

As all patient had visually significant cataract preoperatively, LogMAR of BCVA was also assessed, and this improved from $0.6950 \pm 0.07$ preoperatively to $0.3670 \pm 0.056$, $0.3460 \pm 0.056$, and $0.03370 \pm 0.052$ at 3,6 , and 12 months postoperatively. 

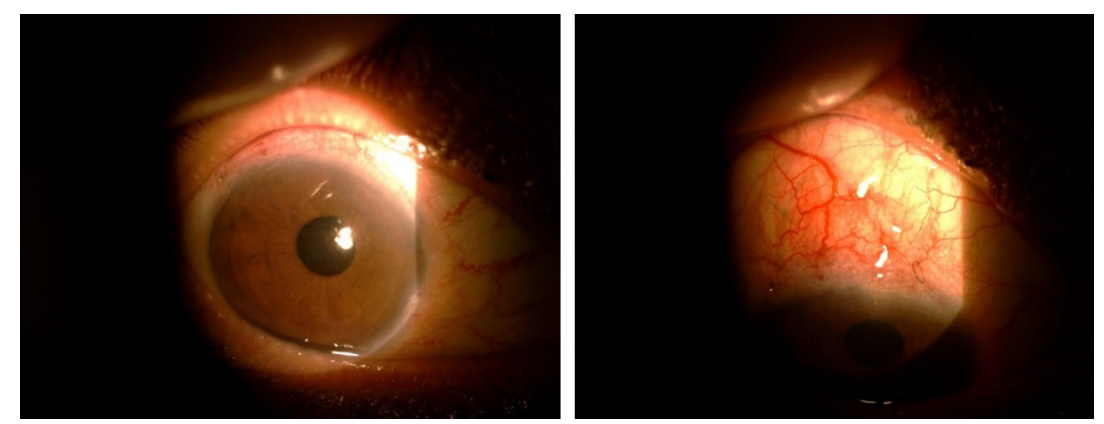

Figure 7 One week after surgery: clear cornea and no bleb.

As regards complications, we had one case with mild hyphema, and conjunctiva was not sutured in one irritable patient. Mild corneal edema was present in 1 eye, mild inflammatory membrane and astigmatism due to tensioning 2DC were seen in 2 eyes. Even in the eye that had recessed conjunctiva, there was no leakage and IOP was controlled, and this means that this surgery does not depend on bleb formation.

In a small case series of Japanese patients receiving canaloplasty or phacocanaloplasty, performed by Fujita et al, ${ }^{12} 11$ eyes of 9 Japanese patients with POAG underwent canaloplasty ( 3 eyes) or phacocanaloplasty ( 8 eyes). The mean preoperative IOP was $23.4 \pm 5.5 \mathrm{mmHg}$ on $2.8 \pm 0.6$ medications. At 1, 3, 6, and 12 months, the mean IOP was $13.7 \pm 2.8 \mathrm{mmHg}, 12.8 \pm 3.5 \mathrm{mmHg}, 14.0 \pm 4.4$, and $15.0 \pm 4.1$, respectively. The mean postoperative medications significantly decreased to $1.2 \pm 0.8(P<0.01)$. A qualified success rate with and IOP $<21,18$, or $16 \mathrm{mmHg}$ with or without medications at 12 months was achieved in $81.8 \%, 54.5 \%$, and $54.5 \%$, respectively, and this is in agreement with our results.

In a multicenter case study conducted by Shingleton et al, ${ }^{13} 54$ eyes that had combined glaucoma and cataract surgery performed by 11 surgeons at 9 study sites were included. The mean baseline IOP was $24.4 \mathrm{mmHg} \pm 6.1$ (SD) with a mean of $1.5 \pm 1.0$ medications per eye. In all eyes, the mean postoperative IOP was $13.6 \pm 3.8 \mathrm{mmHg}$ at 1 month, $14.2 \pm 3.6 \mathrm{mmHg}$ at 3 months, $13.0 \pm 2.9 \mathrm{mmHg}$ at 6 months, and $13.7 \pm 4.4 \mathrm{mmHg}$ at 12 months. Medication use dropped to a mean of $0.2 \pm 0.4$ per patient at 12 months. Surgical complications were reported in 5 eyes $(9.3 \%)$ and included hyphema ( $\mathrm{n}=3,5.6 \%)$, Descemet tear $(\mathrm{n}=1,1.9 \%)$, and iris prolapse $(n=1,1.9 \%)$. Transient IOP elevation of more than $30 \mathrm{mmHg}$ was observed in 4 eyes (7.3\%) 1 day postoperatively.

Although there is agreement with our results, this study was a multicenter one with 11 surgeons who had different surgical skills and learning curves, and also more number of eyes, 54 versus 20 eyes in our study, was studied. In our study, we excluded the cases that had penetration of the anterior chamber or failed cannulation to see the results of the successful phacocanaloplasty.

In retrospective comparative chart review of consecutive cases by Arthur et al, ${ }^{14}$ thirty-seven patients underwent phacoemulsification and 32 patients had phacocanaloplasty. Follow-up was $21.8 \pm 10.1$ versus $18.8 \pm 9.6$ months for phacoemulsification and phacocanaloplasty, respectively $(P=0.21)$. Age (yr) $(74.7 \pm 9.8$ versus $76.1 \pm 8.3, P=0.54)$, sex $(P=81)$, and laser status $(P=0.75)$ were similar between the
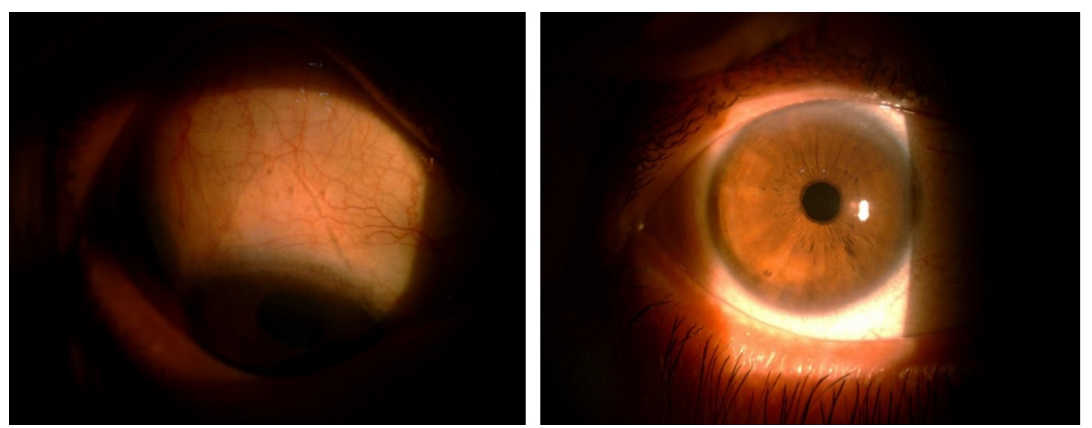

Figure 8 Three months after surgery: coapted sclera flap and no bleb. 


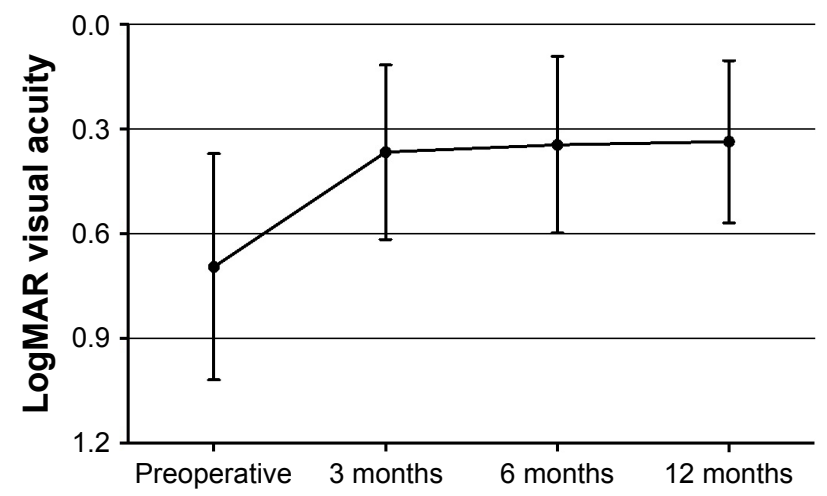

Figure 9 Visual acuity at baseline, 3, 6, and 12 months post-treatment.

groups. Preoperatively, mean \pm SD $\log$ MAR visual acuity ( $0.5 \pm 0.7$ versus $0.5 \pm 0.5, P=0.77)$, IOP $(16.2 \pm 4.6$ versus 18.2 $\pm 5.1, P=0.13)$, and medications used $(1.4 \pm 1.1$ versus $1.3 \pm 0.7, P=0.75$ ) were similar for phacoemulsification and phacocanaloplasty, respectively. At 24-month follow up of phacoemulsification $(n=17)$ and phacocanaloplasty $(n=11)$ mean \pm SD were respectively: $\operatorname{logMAR}$ visual acuity $0.2 \pm 0.2$ versus $0.4 \pm 0.7, P=0.29$; IOP $14.1 \pm 4.0$ versus $12.9 \pm 3.8, P=0.43$; and Meds $1.5 \pm 1.2$ versus $0.3 \pm 0.5, P=0.005$. Rates of successful IOP lowering without medications for phacoemulsification versus phacocanaloplasty at 12 months were $34 \%$ versus $75 \%$, respectively $(P=0.003)$. The studied mean reduction in IOP was higher with the combined procedure, similar to our case series when compared to phacoemulsification alone.

Our case series was prospective and interventional, with patient selection that addressed two significant problems in one surgical session. There is relatively long follow-up of cases (12 months) and low incidence of postoperative complications compared to penetrating glaucoma surgery either performed alone or in combination with phacoemulsification. The main drawbacks are small sample size due to high cost of the procedure, nonrandomized nature of the study, and noncomparative nature with liability to selection bias.

Table 3 Visual acuity at baseline, 3, 6, and 12 months posttreatment

\begin{tabular}{ll}
\hline & $\begin{array}{l}\text { Combined phaco } \\
\text { and canaloplasty } \\
\mathbf{n}=\mathbf{2 0}\end{array}$ \\
\hline Preoperative VA, LogMAR (mean \pm SD) & $0.6950 \pm 0.07$ \\
3 months & $0.3670 \pm 0.056^{*}$ \\
6 months & $0.3460 \pm 0.056^{*}$ \\
12 months & $0.3370 \pm 0.052^{*}$ \\
\hline
\end{tabular}

Notes: $* P=0.0005$. There was no statistically significant difference in LogMAR between 3,6 , and 12 months postoperatively $(P=0.435)$.

\section{Conclusion}

A combination of canaloplasty with phacoemulsification results in a decreased number of glaucoma medications while increasing the complete success rate for IOP-lowering effect without medication. Prospective long-term randomized studies are needed to validate our findings.

\section{Disclosure}

The authors report no conflicts of interest in this work.

\section{References}

1. Radhakrishnan S, Quigley HA, Jampel HD, et al. Outcomes of surgical bleb revision for complications of trabeculectomy. Ophthalmology. 2009; 116:1713-1718.

2. Prasad N, Latina MA. Blebitis and endophthalmitis after glaucoma filtering surgery. Int Ophthalmol Clin. 2007;47:850-897.

3. Greenfield DS, Suner IJ, Miller MP, Kangas TA, Palmberg PF, Flynn HW. Endophthalmitis after filtering surgery with mitomycin. Arch Opthalmol. 1996;114(8):943-949.

4. AGIS Investigators. The Advanced Glaucoma Intervention Study; 8: risk of cataract formation after trabeculectomy. Arch Ophthalmol. 2001; 119:1771-1779.

5. Hylton C, Congdon N, Friedman D, et al. Cataract after glaucoma filtration surgery. Am J Ophthalmol. 2003;135:231-232.

6. Feiner L, Piltz-Seymour JR. Collaborative Initial Glaucoma Treatment Study: a summary of results to date. Curr Opin Ophthalmol. 2003;14: 106-111.

7. Lewis RA, von Wolff $\mathrm{K}$, Tetz M, et al. Canaloplasty: circumferential viscodilation and tensioning of Schlemm's canal using a flexible microcatheter for the treatment of open-angle glaucoma in adults; interim clinical study analysis. J Cataract Refract Surg. 2007;33: 1217-1226.

8. Lewis RA, von Wolff K, Tetz M, et al. Canaloplasty: three year results of circumferential viscodilation and tensioning of Schlemm canal using a microcatheter to treat open-angle glaucoma. J Cataract Refract Surg. 2011;37:682-690.

9. Grieshaber MC, Pienaar A, Olivier J, Stegmann R. Canaloplasty for primary open-angle glaucoma: long-term outcome. Br J Ophthalmol. 2010; 94:1478-1482.

10. Khaimi MA. Canaloplasty using iTrack 250 microcatheter with suture tensioning on Schlemm's canal. Middle East Afr J Opthalmol. 2009; 19:127-129.

11. Poley BJ, Lindstrom RL, Samuelson TW, et al. Intraocular pressure reduction after phacoemulsification with intraocular lens implantation in glaucomatous and non glaucomatous eyes: evaluation of a causal relationship between the natural lens and open angle glaucoma. J Cataract Refract Surg. 2009;35:1946-1955.

12. Fujita K, Kitagawa K, Ueta Y, Nakamura T, Miyakoshi A, Hayashi A. Short-term results of canaloplasty surgery for primary openangle glaucoma in Japanese patients. Case Rep Ophthalmol. 2011;2: 65-68.

13. Shingleton B, Tetz M, Korber N. Circumferential viscodilation and tensioning of Schlemm canal (canaloplasty) with temporal clear corneal phacoemulsification cataract surgery for openangle glaucoma and visually significant cataract: one-year results. $J$ Cataract Refract Surg. 2008;34:433-440.

14. Arthur SN, Cantor LB, WuDunn D, et al. Efficacy, safety, and survival rates of IOP-lowering effect of phacoemulsification alone or combined with canaloplasty in glaucoma patients. J Glaucoma. 2014;23: 316-320. 


\section{Publish your work in this journal}

Clinical Ophthalmology is an international, peer-reviewed journal covering all subspecialties within ophthalmology. Key topics include: Optometry; Visual science; Pharmacology and drug therapy in eye diseases; Basic Sciences; Primary and Secondary eye care; Patient Safety and Quality of Care Improvements. This journal is indexed on

Submit your manuscript here: http://www.dovepress.com/clinical-ophthalmology-journal
PubMed Central and CAS, and is the official journal of The Society of Clinical Ophthalmology (SCO). The manuscript management system is completely online and includes a very quick and fair peer-review system, which is all easy to use. Visit http://www.dovepress.com/ testimonials.php to read real quotes from published authors. 